\title{
LR PROCESS EXPERT - COST-EFFICIENT AND MODULAR PROCESS AUTOMATION EXPERT CLASS SYSTEM FOR LONG ROLLING PLANTS*
}

\author{
Paul Riches ${ }^{1}$
}

Piotr Czupryna ${ }^{2}$

\begin{abstract}
LR Process Expert is a cost-efficient process automation system for all types of long rolling mills, including, rod, bar, section, rail, reversing mills, and flat blocks. The system performs a wide range of tasks for optimized operation - from suggesting pass schedules to calculating and downloading optimized mill settings according to the availability of rolls. LR Process Expert also monitors and collects data from the raw material stage through to the finished product. It delivers valuable mill reports to the operator staff and management so they can easily assess a plant's performance and optimize production. Defined Key Performance Indicators (KPIs) help to assess a plant's performance; this system is not limited to one plant but can enable plant-toplant performance at corporate level.

Keywords: Long rolling; Key performance indicators (KPIs); Expert system; Mill management; Production data analysis; Roll shop management; Pass schedule handling; Delays classification; Long rolling mill production scheduling.
\end{abstract}

1 Global Head of Electrics \& Automation, Primetals Technologies USA LLC Long Rolling, Worcester MA, USA.

2 CEO Primetals Technologies, Poland. 


\section{INTRODUCTION}

"You cannot manage what you do not measure" is an eternal principle of management. Until you can measure your processes, you have no idea whether they are getting better or worse. You can head towards improvement, but you do not have the data to analyze what is changing and verify that it is changing in the right direction.

Today's steel producers face the dual challenge of ensuring on-time delivery and ever demanding product requirements while also running a lean operation. Everyday management of a long rolling plant requires continuous optimization of both operating and business practices. By measuring key performance indicators (KPIs) and comparing them against set targets or world standards, this can be achieved. To successfully accomplish this, you need both reliable data and accurate reporting.

Modern superimposed IT systems (like SAP / ERP class systems) require more and more data to be collected and analyzed. Quality and effectiveness standards require various KPIs to be calculated, monitored and presented against known standards.

Additionally to this the end, customers are requiring that specific data related to their particular product be collected. They can ask for full production history to ensure that the end product received meets their requirements.

Today more and more long rolling customers require powerful reporting tools, not only for collecting data but also a flexible system to analyze the data in different ways.

The long products market is sensitive to market trends, changes in the market and cost fluctuations. In such a dynamic market, long rolling producers need to monitor both their operations and processes in order to be able to dynamically adapt to market trends.

Monitoring the mean time between failures (MTBF), calculating a wide variety of KPIs, monitoring and scheduling plant maintenance and planning shifts and crews all are not only new market demands but a must for the business. In today's market every single euro or even cent counts in the operation of the long rolling business. An effective operation is paramount.

To meet these requirements, Primetals Technologies has developed a comprehensive system, the LR PROCESS EXPERT. This solution answers the market demands, is flexible, modular, cost effective and is easily adapted to monitor any type of long rolling plant or plants.

\subsection{A Modern Platform System}

- Multiclient environment with centralized business and process functions

- MVC (Model View Controller) design pattern to separate operator actions and centralize process functions and logic

- Scalability: easy to add new clients

- Capable of working with different communication protocols and partners: TCP/IP, OPC read/write, external datatabases, importing files (for example excel, CSV format), webservices

- Dedicated components to serve different purposes (flexible system structure from project to project)

- Regionalization - data presentation reflects local culture (language, units), possible to switch online local and default language

- Report generation (automatic, on event or time based) 
- Redundancy, fail safe operations

- Corrective actions for all important operations available for operators

- All measurements and analysis tools in one system, not "hidden" in different departments

- Data available to all personnel, from the operators, maintenance, management to corporate level

Nowadays modularity, flexibility and user friendly interfaces are the mainstream trends for such systems.

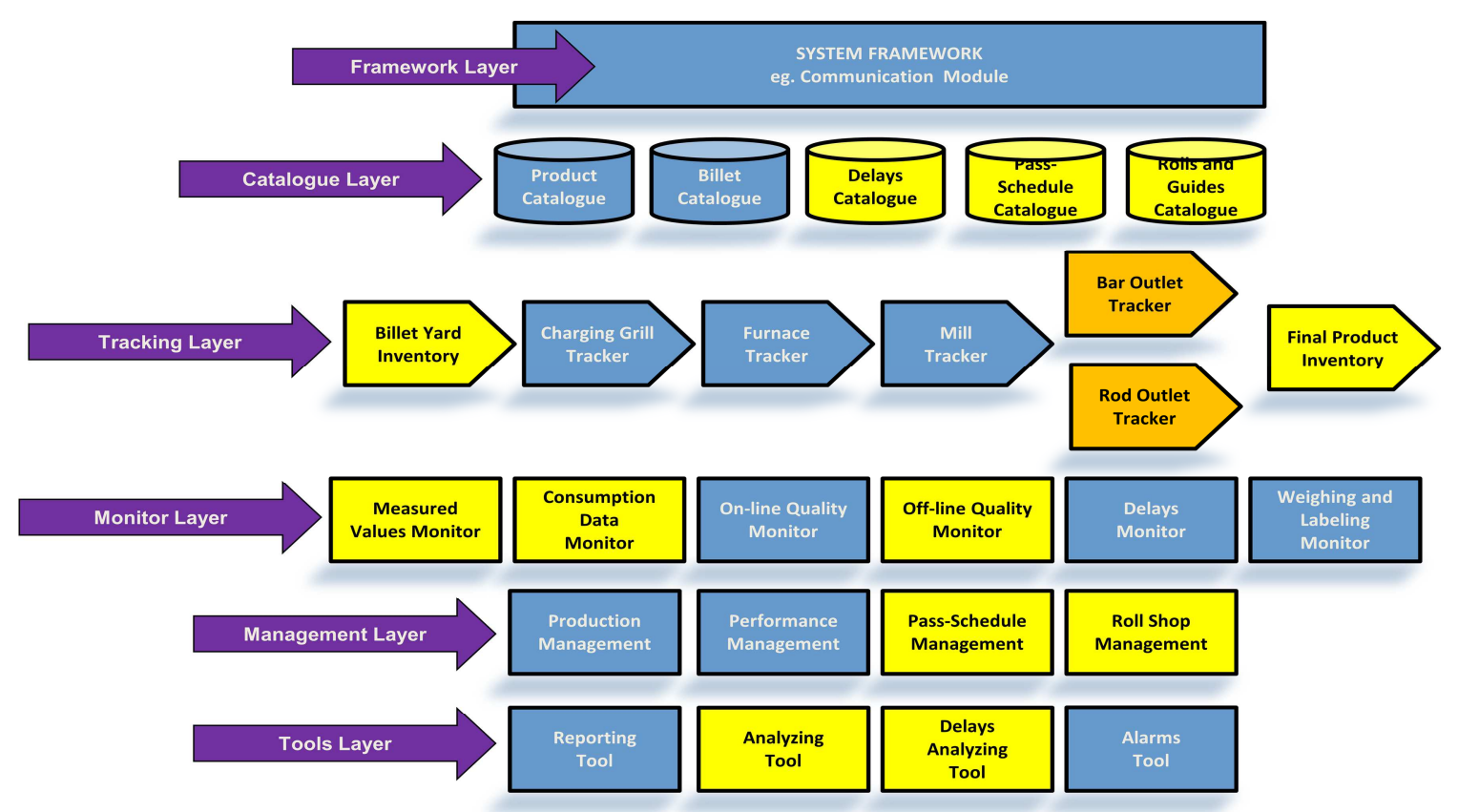

Figure 1. Primetals Technologies LR Process Expert Modules Overview

\section{MATERIAL AND METHODS}

\subsection{System Modules}

Primetals Technologies LR Process Expert provides a modular range of basic functions for managing orders, assets and qualities. This can be supplied as a full system or supplied with the individual modules that a plant requires. If individual modules are supplied, additional modules can be integrated at a later date, so customers can invest at their own pace.

The basic technological modules, based on extensive experience in the long rolling industry, include:

- Production sequencing

- Heat data management

- Raw material assignment

- Automatic mill setup data management and control

- Material tracking

- Data processing/archiving

- Plant monitoring

- Pass Scheduling

- Weighing and tagging

- Analysis and report manager 
- Delay management

- Online system help

- User-friendly integrated HMI

- Definition of KPIs (Key performance indicators and powerful Condition Monitoring)

- Genealogy module

- QUALITY On Line monitor

Optional modules of Primetals Technologies LR Process Expert:

- Interface to MES - Manufacturing Execution System (L3) and other IT systems

- Production program optimization

- Roll and guide management

- Melt shop interface

- Maintenance tracking and guide manager

- Offline sample and laboratory interface generation of QA certificates

- Billets and final product yards inventory

\subsection{Process Automation (L2) - Primetals Technologies LR Process Expert}

Consolidation in the global steel industry is bringing new challenges to the task of managing several plants in different locations and countries. Overall business performance is controlled by business management, but plant performance is achieved at the mill floor operations level. Due to constraints in technology and cultural limitations, there has historically been a weak link between business management and production management in long product rolling mills and also between the company's different work centers (mill to mill).

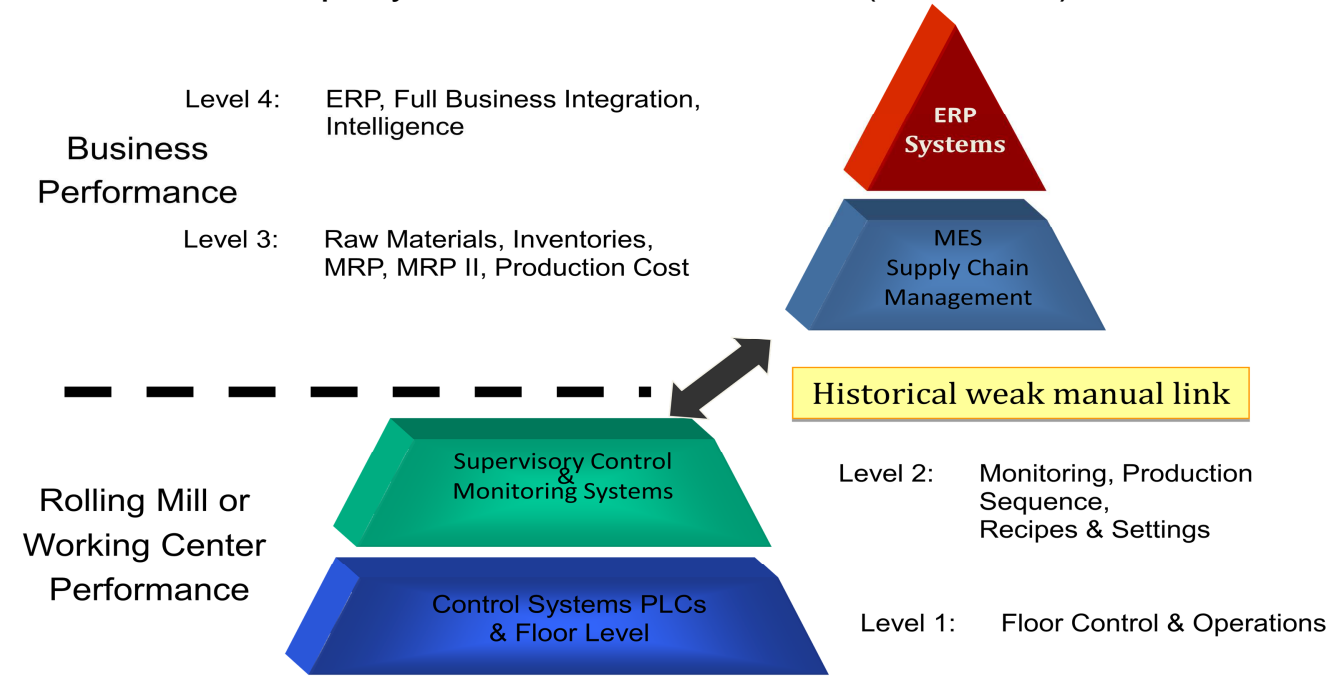

Figure 2. Business Performance Levels as per ISA 95

\subsection{A Bridge to the Future - Topology of Mill Production Control}

In accordance with ISA 95, there are four levels where all business performance and plant data is collated and reviewed. The four levels are shown in Figure 2, which also shows the levels that are typically driven and managed by the business side and those that are driven by the production or work centers. 


\section{9th Industrial $\Pi$ and Automation}

With the technological evolution of PLC control, data collection and production monitoring devices, as well as the development of fully integrated ERP systems, we can now integrate and bridge the four levels. This bridge has been missing in the past, and it is the critical advantage of the Primetals Technologies LR Process Expert system.

The Primetals Technologies LR Process Expert solution domain extends from the Level 1 basic automation system that provides all basic rolling data and information to the MES level with its product genealogy relationships, production planning and reporting.

The Primetals Technologies LR Process Expert collects the data from Level 1 with all management data and presents the resulting information in a standardized format to meet all specific mill or company requirements, including reporting, operations and mill operation tracking. Complex KPIs from the operational perspective are prepared and displayed, with tools provided to analyze and locate areas of opportunity as well as drive continuous improvement.

\subsection{Description of the Main System}

\subsubsection{Material tracking}

Dependent upon the mill configuration, the system can track from the Billet Yard to the Charging Grill, through the Furnace, Mill or Bar Outlets / Coil Outlets, Bar Handling Area / Coil Handling Area, up to the Finished Product Yard.

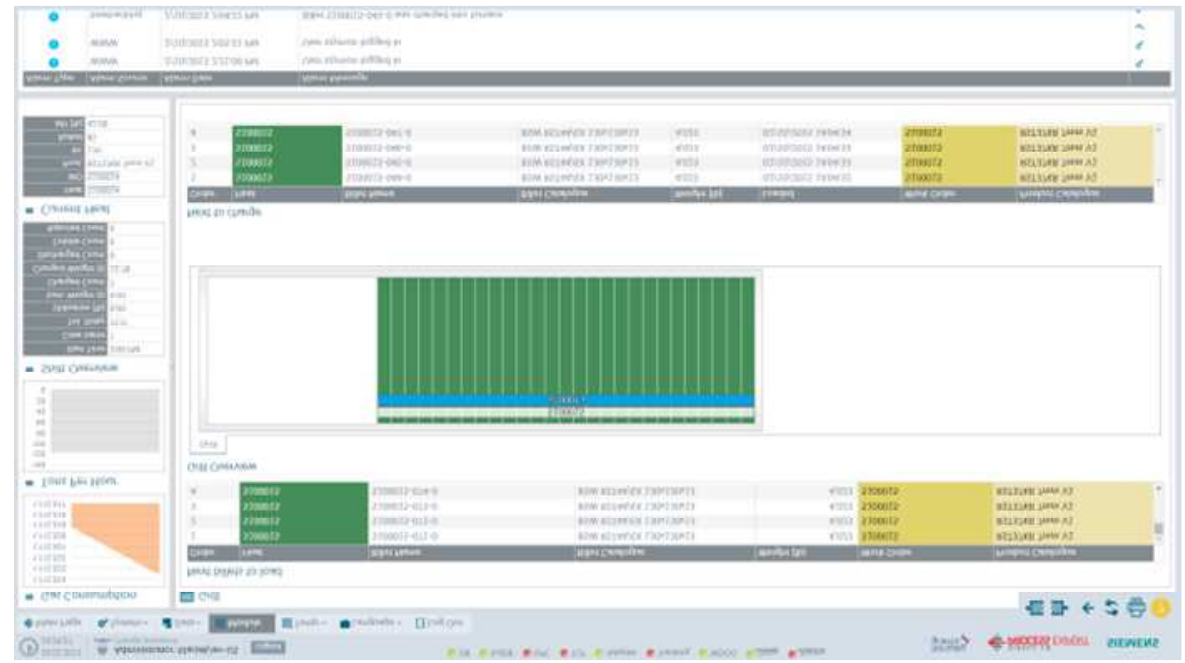

Figure 3. Charging Grill Tracking

\subsubsection{Data Analysis and Reporting}

Primetals Technologies LR Process Expert has a flexible data analysis module that allows the end user to view data from different perspectives to ensure the data can be properly analyzed and presented. 


\section{9th Industrial II and Automation}

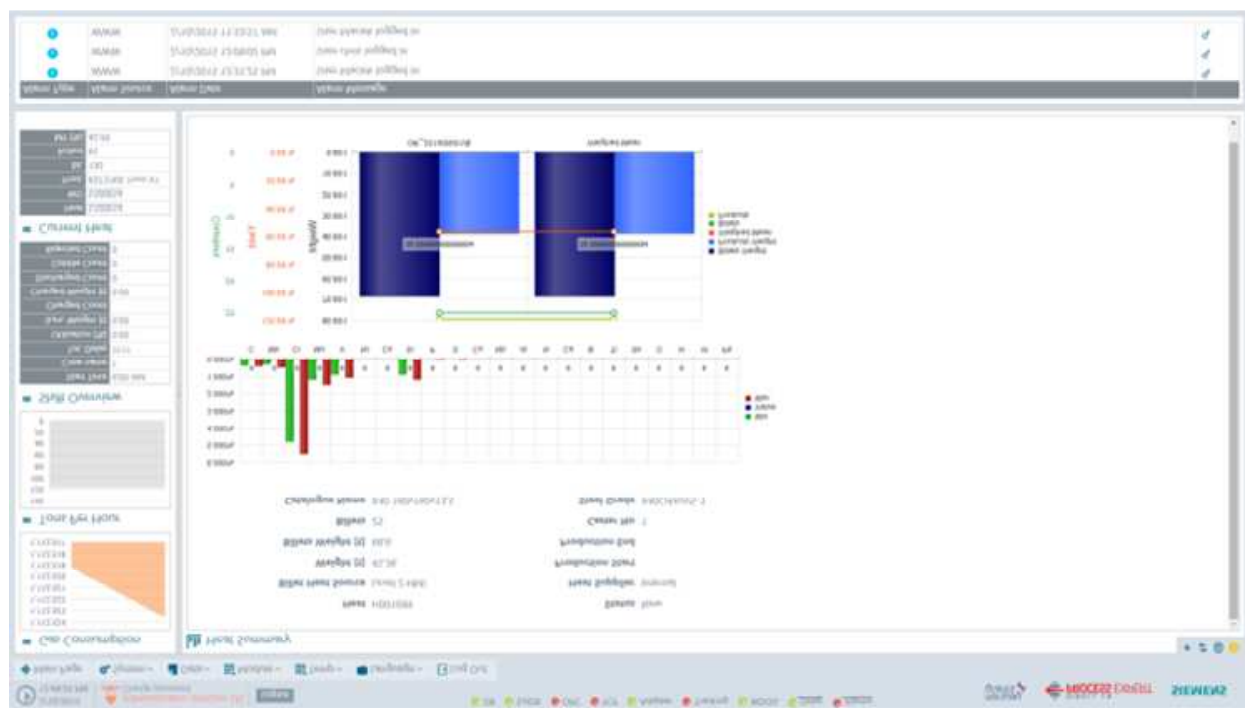

Figure 4. Example of Report - Heat Report

Analysis tools include the following:

- Gas Consumption

- Energy Consumption

- Weight tons per time in different views (hours, months, year, crew, shift, etc.)

- Production Throughput

- Heat Summary

- Mill Utilization report in different views (per shift, per time, per crew, etc.)

- Cobble analysis

- Delays Analysis

- Rolls History

The system is flexible, allowing other tools to be added easily and efficiently if the data is available to be collected.

\subsubsection{Production Management}

The system also manages the production schedule, binding given orders to particular heats and billets, defining production parameters and batches. The storage module suggests the best matching billets from the billet yard to assign to the heat. Most of the operations are started based on operator input but certain feedback from the tracking module is registered and processed as well. The main task of the operator is to build a schedule based on received orders and the released batch for production. The batch is then merged with the existing production schedule and subsequent events from the tracking module are collected to update the status of the batch and schedule. After the "heat end" signal, the module calculates metallic yield and other KPIs based on the collected data. Emergency corrective actions like adding or removing billets to/from production schedule are also supported.

The system can receive and create the production orders manually or by interface with other system (with $\mathrm{xml}$ or *.xls files).

\subsubsection{Pass Schedule Handling}

The system provides functionality which allows management of the Pass Schedules, updates to the schedule and assignment to a specific product. 
All of this data can be inserted, corrected and redefined manually. Additionally, all of this data can be received from the Level 3 or from special Roll Pass design tools (e.g., Primetals Technologies LR Roll Master).

\subsubsection{Rolls and Guides Management Functionality}

Roll set data consists of:

- Roll data (assigned to each stand)

- Groove data (assigned to each roll)

The main functions of the roll shop management module are:

- Configures mill: Define the type of mill, stands, rolls, mounting, status, predicted pass and / or roll change

- Maintains (input, update, delete) roll/roll set data

- Generates machining work orders for rolls

- Reports when WO is completed

- Roll Turning Labor and reports

- Measures Tons accumulated per roll

- Allows Pulpit to see rolls for next rolling campaign

- Maintains (input, update, delete) pass schedule data

- Supports roll change/mount/dismount/scrap of roll sets via Level 2 dialogs

- Monitors usage during production by adding rolled length (eventually rolled tonnage) of produced material to rolls and grooves. If rolled weight exceeds a predefined limit for a groove, then an alarm is output by the Level 2 system to inform the operator that a roll change is necessary.

- Supports treatment of rolls. After grinding, operator must input the new of roll diameter via Level 2 dialog.

- Provides a standard set of dialogs and reports to get information about the history of rolls/roll sets

- Monitors roll inventory and can either order dynamically when stock reaches a preassigned level or alert the roll shop manager that new roll sets are required.

\subsubsection{Primetals Technologies LR Roll Master}

The Roll Master extends the automation with a dedicated computer-based pass schedule calculation. Rolling requirements for new products and grades can be precalculated and checked before they enter the "real world" of a plant, greatly reducing the risk of a prolonged start up on a new product or size, or of a long trial-and-error process. Depending on the specific mill design, LR Roll Master also analyzes plant and drive limits to create and optimize pass schedules. Optimization is based on a built-in material database that contains, among other things, more than 200 steel grades and alloys as well as rolling temperature models.

\section{BENEFITS}

\subsection{Highest Product Quality}

Long rolling producers are challenged to provide products that meet ever-increasing quality standards. Older or outdated automation systems cannot achieve the quality control required for process production standards. Anything less than the most modern tracking methods and process handling equipment risks compromising productivity, metallurgical properties, cut length, packaging quality, and the overall 
product quality. By monitoring the process parameters, metallurgical properties collected online and offline, billet to billet, heat to heat and product to product, any quality issues can be resolved and identified early and cost effectively. Quality improvements can be easily identified.

\subsection{Maximum Plant Efficiency}

While demands on products and processes are always increasing, steel producers are faced with limitations caused by inadequate automation technology, old equipment and a lack of spare parts.

By monitoring the downtime and assigning the loss of time to a specific part, crew or maintenance area, the problem areas causing the main downtimes can be identified. Through an understanding this and identifying the areas or parts, the mill utilization can be improved, spare parts can be ordered with confidence and investment planning can be scheduled with a strong argument for the return of investment.

\subsection{Flexibility in Changing Markets}

Production lines must be flexible enough to react to market requests, and be able to produce the steel grades that end users demand - this can be achieved using the best-quality electrical equipment and flexible production setting and tracking systems.

\section{CONCLUSION}

\subsection{Advantages of the Primetals Technologies LR Process Expert:}

- High cost efficiency based on a modular client-server architecture

- Exactly the data security you need with a system that can be expanded to a high level of redundancy

- Easy upgrades in running plants and for new applications by "grabbing" current data from any type of PLC

- High process transparency through in-depth analysis and reporting functions based on real-time data tracking

- Fast management overview with powerful reporting functions

- Easy comparison of your facilities all over the world - with agreed KPIs

- High flexibility for any kind of long rolling plant

- Proven track record more than 25 installations worldwide

Primetals Technologies LR Process Expert is part of the Primetals Technologies electrics and automation portfolio for the long rolling industry. It can be supplied stand-alone or seamlessly integrated into Primetals Technologies Long Rolling automation concepts. 